\title{
Mean Diffusional Kurtosis in Patients with Glioma: Initial Results with a Fast Imaging Method in a Clinical Setting
}

\author{
A. Tietze, M.B. Hansen, L. Østergaard, S.N. Jespersen, R. Sangill, T.E. Lund, M. Geneser, M. Hjelm, and B. Hansen
}

\begin{abstract}
BACKGROUND AND PURPOSE: Diffusional kurtosis imaging is an MR imaging technique that provides microstructural information in biologic systems. Its application in clinical studies, however, is hampered by long acquisition and postprocessing times. We evaluated a new and fast ( 2 minutes 46 seconds) diffusional kurtosis imaging method with regard to glioma grading, compared it with conventional diffusional kurtosis imaging, and compared the diagnostic accuracy of fast mean kurtosis (MK') to that of the widely used mean diffusivity.
\end{abstract}

MATERIALS AND METHODS: MK' and mean diffusivity were measured in the contrast-enhancing tumor core, the perifocal hyperintensity (indicated on T2 FLAIR images), and the contralateral normal-appearing white and gray matter of 34 patients (22 with high-grade and 12 with low-grade gliomas). MK' and mean diffusivity in the different tumor grades were compared by using a Wilcoxon rank sum test. Receiver operating characteristic curves and the areas under the curve were calculated to determine the diagnostic accuracy of MK' and mean diffusivity.

RESULTS: $M K^{\prime}$ in the tumor core, but not mean diffusivity, differentiated high-grade from low-grade gliomas, and $M^{\prime}$ differentiated glioblastomas from the remaining gliomas with high accuracy (area under the curve $\mathrm{MK}^{\prime}=0.842 ; P_{\mathrm{MK}^{\prime}}<.001$ ). MK' and mean diffusivity identified glioblastomas in the group of high-grade gliomas with similar significance and accuracy (area under the curve MK' $^{\prime}=0.886$; area under the curve $\left.e_{\text {mean diffusivity }}=0.876 ; P_{\mathrm{MK}^{\prime}}=.003 ; P_{\text {mean diffusivity }}=.004\right)$. The mean $\mathrm{MK}^{\prime}$ in all tissue types was comparable to that obtained by conventional diffusional kurtosis imaging.

CONCLUSIONS: The diffusional kurtosis imaging approach used here is considerably faster than conventional diffusional kurtosis imaging methods but yields comparable results. It can be accommodated in clinical protocols and enables exploration of the role of MK' $^{\prime}$ as a biomarker in determining glioma subtypes or response evaluation.

ABBREVIATIONS: $\mathrm{AC}=$ astrocytoma; $\mathrm{DKI}=$ diffusional kurtosis imaging; $\mathrm{GBM}=$ glioblastoma multiforme; $\mathrm{HGG}$ = high-grade glioma; $\mathrm{LGG}=$ low-grade glioma; $M D=$ mean diffusivity; $M K=$ mean kurtosis; MK' $^{\prime}$ fast mean kurtosis; $N A G M=$ normal-appearing gray matter; $N A W M=$ normal-appearing white matter

D iffusion-weighted and diffusion tensor imaging are currently used in patients with cerebral glioma as presurgical imaging tools for the World Health Organization grading system, neuro-

Received November 18, 2014; accepted after revision January 24, 2015.

From the Department of Neuroradiology (A.T., L.Ø., M.G., M.H.), Aarhus University Hospital, Aarhus, Denmark; Center of Functionally Integrative Neuroscience and MINDLab, Institute of Clinical Medicine (A.T., M.B.H., L.Ø., S.N.J., R.S., T.E.L., M.G., B.H.) and Department of Physics and Astronomy (S.N.J.), Aarhus University, Aarhus, Denmark.

This work was supported by the Danish Ministry of Science, Technology and Innovation's University Investment Grant (MINDLab). B.H. acknowledges support from NIH 1R01EB012874-01.

Please address correspondence to Anna Tietze, MD, Department of Neuroradiology, Aarhus University Hospital, Nørrebrogade, DK-8000 Aarhus, Denmark; e-mail: annatietze@gmail.com

- Indicates open access to non-subscribers at www.ajnr.org

三 Indicates article with supplemental on-line table

http://dx.doi.org/10.3174/ajnr.A4311 navigation, and response evaluation during radiochemotherapy or antiangiogenic treatment. ${ }^{1}$ DTI and DWI approximate the displacements of diffusing water molecules by a Gaussian distribution as if water molecules were moving unrestricted in all directions. However, it is well established that the diffusion signal from cerebral water molecules for moderate-to-high values of the magnetic gradient field is not described accurately by the standard monoexponential decay as a function of the diffusion weighting (b-value). ${ }^{2}$

Diffusional kurtosis imaging (DKI) is a recently described MR imaging technique that aims to provide additional microstructural information by extending the DTI model to incorporate fourth-order gradient field terms in the diffusion signal. ${ }^{2}$ In this higher-order description of diffusion signal decay, the dimensionless kurtosis term describes the degree of deviation from the Gaussian distribution of spin displacements along the axis of observation. When averaged over all directions, the mean kurtosis (MK) is obtained. ${ }^{2}$ The deviation from free (Gaussian) diffusion 
is generally assumed to be caused by tissue microstructure, and $\mathrm{MK}$ is therefore interpreted as a general microstructural marker. ${ }^{3}$ Use of the DKI model may be beneficial in 2 ways: 1) the diffusion tensor is indirectly corrected through the higher-order terms, ${ }^{4}$ and 2) the additional microscopic diffusion characteristics may be used for clinical purposes. Compared with gray matter or edematous tissue, white matter and malignant tumors are characterized by higher architectural complexity that results from cell membranes, organelles, axons, or vascular structures impeding proton diffusion and leading to higher non-Gaussianity and increased MK. ${ }^{5}$

Gliomas are a heterogeneous group, categorized as low-grade glioma (LGG) and a high-grade glioma (HGG) with different histopathologic features, such as cellularity or the presence or absence of necrosis and neoangiogenesis. This heterogeneity is likely to be reflected in MK alterations, and promising first studies have reported $\mathrm{MK}$ as an imaging-based means of presurgical glioma grading. ${ }^{6,7}$ Larger studies and the more widespread use of DKI, however, have been held back by relatively long acquisition times that result from a model requirement for data to be obtained along several directions at each of many b-values. ${ }^{8}$ Such protocols are clearly incompatible with the daily routines in busy clinical imaging departments and are often not tolerable by critically ill patients.

We recently proposed a kurtosis method ${ }^{9,10}$ that is much faster than traditional kurtosis-acquisition schemes, ${ }^{2,4}$ in terms of both acquisition and postprocessing times, and on the basis of this method we present here our first clinical results in 34 patients with glioma. We correlated MK values with histopathologic grades and compared them with those from the literature. Moreover, we compared the diagnostic accuracy of MK with that of the widely used mean diffusivity (MD), calculated in our case as part of the fast-MK method.

\section{MATERIALS AND METHODS \\ Patients}

Thirty-five patients with cerebral gliomas were included in this retrospective study. A waiver from the local ethics committee was granted, because DKI was acquired without increasing the scan time by modification of the diffusion-weighted sequence that is a part of our routine clinical protocol. Apart from steroids in some patients with glioblastoma multiforme (GBM), these patients did not receive any treatment at the time of imaging. One patient had to be excluded because of motion artifacts on conventional sequences so severe that reliable tumor outlining could not be performed. The diagnosis of glioma (22 HGGs, 12 LGGs) was determined by biopsy or resection in 30 cases. Three of the remaining 4 cases without histopathology results were considered to be LGG and 1 was considered to be GBM; these diagnoses were based on the clinical behavior and imaging characteristics on conventional MR imaging. Two of 34 patients, both with LGG, had undergone surgery many years before being included in this study and were re-imaged because of disease recurrence. Included were 15 patients with GBM, 5 with a grade III astrocytoma (AC), 2 with a grade III oligodendroglioma, and 12 with an LGG (3 with a proven grade II oligodendroglioma). One tumor was classified as a grade II oligodendroglioma with a minor astrocytic component and was subsequently included in the group of those with a grade II oligodendroglioma.

\section{Imaging}

MR imaging was performed on a Skyra 3T system (Siemens, Erlangen, Germany) with a standard 20-element head coil. The MR imaging protocol consisted of 3D T1-weighted images before and after intravenous contrast $(0.1 \mathrm{mmol} / \mathrm{kg}$ gadoterate meglumine; TR, $2300 \mathrm{~ms}$; TE, $3.8 \mathrm{~ms}$; voxel size, $1 \times 1 \times 1 \mathrm{~mm}^{3}$; FOV, $256 \times$ $256 \mathrm{~mm}^{2}$; acquisition time, $349 \mathrm{~s}$ ) and axial precontrast T2 FLAIR images (TR, $9000 \mathrm{~ms}$; TE, $117 \mathrm{~ms}$; TI, $2500 \mathrm{~ms}$; voxel size, $0.7 \times$ $0.7 \times 3 \mathrm{~mm}^{3}$; FOV, $230 \times 220 \mathrm{~mm}^{2}$; acquisition time, $326 \mathrm{~s}$ ). Axial DKI was performed before intravenous contrast (TR, 10,300 ms; TE, $100 \mathrm{~ms}$; voxel size, $2 \times 2 \times 2 \mathrm{~mm}^{3}$; FOV , $196 \times 196 \mathrm{~mm}^{2}$; number of excitations, 1 ; sections, 60 ; acquisitions, 3 [one along each of the $\mathrm{x}^{-}, \mathrm{y}^{-}$, and $\mathrm{z}$-directions] with $b=1000 \mathrm{~s} / \mathrm{mm}^{2}, 9$ different directions [as specified in Hansen et $\mathrm{al}^{9}$ ] with $b=2500$ $\mathrm{s} / \mathrm{mm}^{2}$, and 1 acquisition with $b=0 \mathrm{~s} / \mathrm{mm}^{2} ; 13$ diffusionweighted images in total). The acquisition time for DKI was 166 seconds.

\section{MR Imaging Data Analysis}

In the framework of the fast kurtosis sequence, ${ }^{9} \bar{W}$, the mean of the kurtosis tensor $W$, is computed, and it was shown to be very similar numerically to the traditional MK. Here, we refer to it as $\mathrm{MK}^{\prime}$ for convenience. $\mathrm{MK}^{\prime}$ is calculated from 13 diffusionweighted images: $1 b=0$ scan for normalization, 3 images at $b=1000 \mathrm{~s} / \mathrm{mm}^{2}$ along each of the $\mathrm{x}^{-}, \mathrm{y}^{-}$, and $\mathrm{z}$-directions, and 9 images at $b=2500 \mathrm{~s} / \mathrm{mm}^{2}$ along the 9 directions defined in Hansen et al. ${ }^{9}$ From these data, a robust estimate of MD can also be achieved with the kurtosis term taken into account in its calculation, which was shown to improve MD estimates ${ }^{4}$ and implemented as proposed in Jensen et al. ${ }^{11}$

Postprocessing of the DKI data, including the reslicing and co-registration steps, were performed by running modules developed in-house in SPM8 (http://www.fil.ion.ucl.ac.uk/spm/ software/spm8) and Matlab (MathWorks, Natick, Massachusetts).

Contrast-enhanced T1-weighted images were resliced to T2 FLAIR. A neuroradiologist, blinded for the histopathologic diagnosis (A.T., 5 years of experience), outlined the tumor core (contrast-enhancing part), the perifocal hyperintensity on T2 FLAIR (hereafter termed FLAIR mask), and regions of normal-appearing white and gray matter (NAWM and NAGM, respectively) in the contralateral centrum semiovale and thalamus (Fig 1). In cases of a nonenhancing lesion, only T2 FLAIR was used to define the tumor. Normal vessel structures, necrotic areas, and potential blood products were avoided, guided by conventional sequences. Tumor core, FLAIR masks, contralateral NAWM masks, contrast-enhanced T1, and T2 FLAIR images were subsequently co-registered and resliced to the localization of the DKI data. The co-registration step was checked visually by using SPM8.

The duration of the DKI sequence is quite short (166 seconds); hence, it was deemed unnecessary to perform motion correction of the individual volumes. The validity of this strategy was verified 


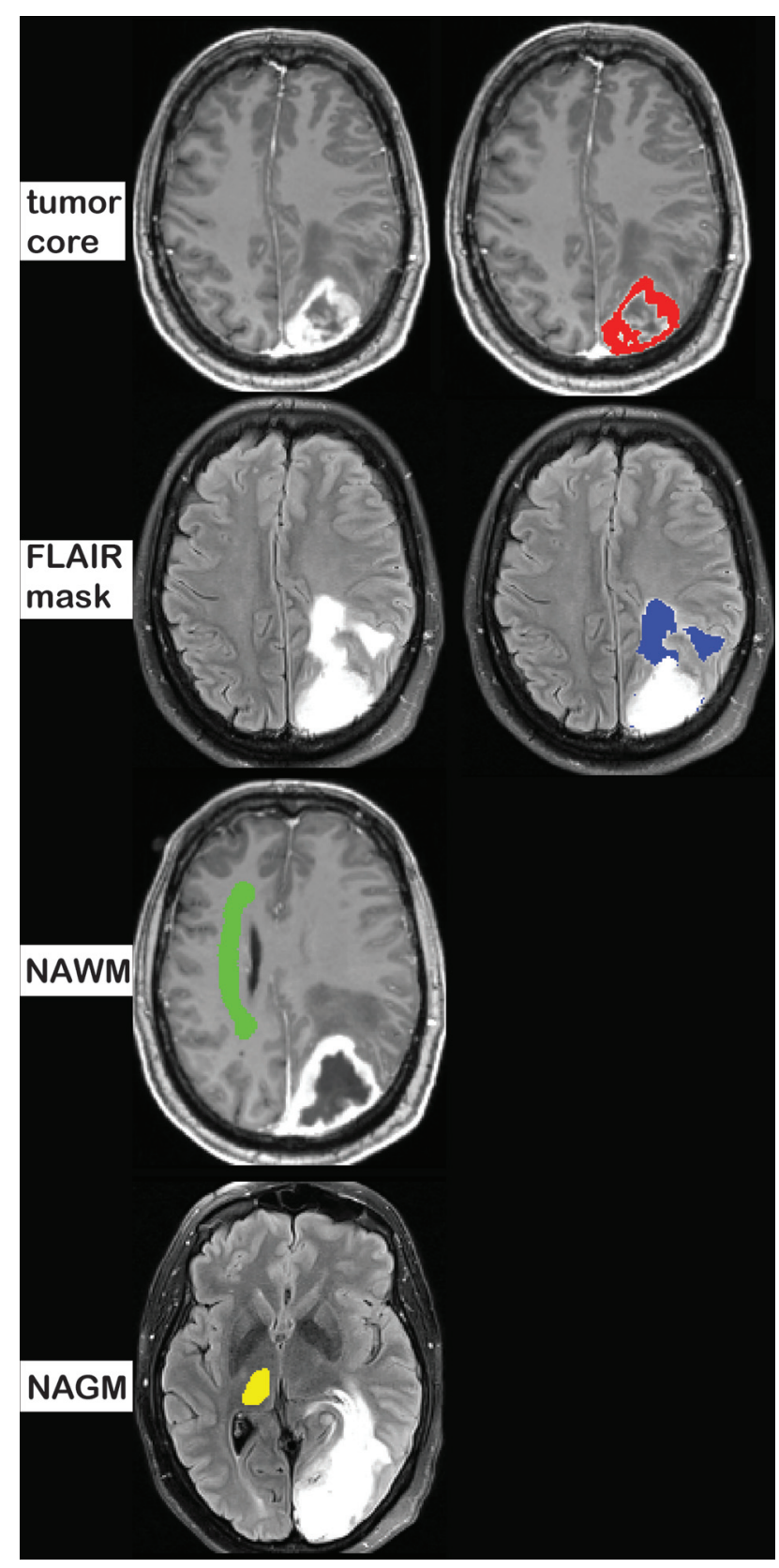

FIG 1. The tumor core was defined as the contrast-enhancing part on postcontrast T1-weighted images, and the FLAIR masks were defined as hyperintense regions on T2 FLAIR images (minus the tumor core and necrosis, if present). In cases of nonenhancing tumors, hyperintensity areas on T2 FLAIR images were defined as the tumor core (the tumor core and edema were identical regions). The NAWM was outlined in the centrum semiovale of the contralateral hemisphere (on $\geq 4$ consecutive sections). The NAGM was defined in the contralateral thalamus (on $\geq 3$ sections).

in all cases by visual inspection of all diffusion images. To remove possible spurious voxels, the DKI data were up-sampled by a factor of 2 in all directions by using linear interpolation and subsequently smoothed by a $2 \times 2 \times 2-\mathrm{mm}^{3}$ kernel. The spatial distortions introduced in the images by eddy currents were compensated to some extent by using a double spin-echo sequence, which is currently the best clinically viable solution (it does not add additional time to the scan sequence). The postprocessing of a single DKI dataset took 3-5 seconds.

\section{Statistical Analysis}

Statistical analysis was performed by using R Studio (http://rstudio. org/download/desktop) and Matlab. Voxelwise values of MD and $\mathrm{MK}^{\prime}$ values were extracted from the 4 regions. The $\mathrm{MK}^{\prime}$ and MD values were normalized by calculating the ratio of tumor core values and the individual mean values obtained in the contralateral NAWM (termed $n M K^{\prime}$ and $\mathrm{nMD}$, respectively). The same was done for values in the FLAIR masks. The mean values in all the regions were computed. To evaluate data variations of $\mathrm{MK}^{\prime}$ and $\mathrm{MD}$, we calculated the coefficient of variation in pathologic tissue in contralateral normal-appearing tissue (NAWM and NAGM). A paired $t$ test was performed to assess whether variations of $\mathrm{MK}^{\prime}$ and MD were significantly different. A Wilcoxon rank sum test was used to compare mean $\mathrm{MK}^{\prime}$ and $\mathrm{MD}$ values between tumor types and grades. The statistical threshold for significant discrimination was set to a $P$ value of $\leq .05$. Receiver operating characteristic curves were constructed to determine the diagnostic performance of $\mathrm{MK}^{\prime}, \mathrm{MD}, \mathrm{nMK}$, and $\mathrm{nMD}$. The area under the receiver operating characteristic curve was used to measure the accuracy, and $P$ values were used to measure strength. Moreover, logistic regression was performed to investigate whether the combination of $\mathrm{MK}^{\prime}$ and MD increased the area under the curve and thereby the diagnostic performance. Finally, the sensitivity and specificity for discriminating HGG, LGG, and specific tumor grades were determined.

\section{RESULTS}

Typical examples of an LGG (grade II AC, upper panel $A$ ) and an HGG (GBM, lower panel $B$ ) are shown in Fig 2. Note the areas with increased $\mathrm{MK}^{\prime}$ and decreased $\mathrm{MD}$ in the GBM, whereas $\mathrm{MK}^{\prime}$ is low and MD is high in the grade II AC case.

Nonnormalized and normalized mean values of $\mathrm{MK}^{\prime}$ and $\mathrm{MD}$ in the contrast-enhancing tumor core (or the hyperintense area on T2 FLAIR, for nonenhancing tumors) for the different tumor types and grades are shown in Fig 3. Median values for tumor types and grades along with measurements in the contralateral NAWM and NAGM are given in the On-line Table. The data variation in NAWM was significantly larger $(P<.001)$ for MD (coefficient of variation $=0.122 \pm 0.019)$ than for MK' (coefficient of variation $=0.093 \pm 0.013$ ), and MD values varied slightly less (coefficient of variation $=0.163 \pm 0.058)$ than the $\mathrm{MK}^{\prime}$ values (coefficient of variation $=0.189 \pm 0.043)$ in NAGM $(P=.036)$.

The mean value of $\mathrm{MK}^{\prime}$ in the tumor core was significantly higher in HGGs than in LGGs. nMK' was increased in HGGs, but it was with a $P$ value of .058 , which is above the threshold for statistical significance. MD and nMD were not significantly different between the HGGs and LGGs. $\mathrm{MK}^{\prime}$ and $\mathrm{nMK}^{\prime}$ were increased, and MD and nMD were decreased in GBMs when comparing them with the remaining HGGs or with the entire study group consisting of HGGs and LGGs (all $P<.05$ ). In the group of HGGs, grade III AC cases were identified by $\mathrm{MK}^{\prime}, \mathrm{MD}, \mathrm{nMK}^{\prime}$, and nMD. It was not possible to discriminate groups by values in the FLAIR masks. All $P$ values are reported in Table 1.

The receiver operating characteristic curves for the discrimination between HGGs and LGGs showed higher areas under the curve for $\mathrm{MK}^{\prime}$ and $\mathrm{nMK}^{\prime}$ than for MD and $\mathrm{nMD}$ in the tumor core. The areas under the curve for the differentiation between 


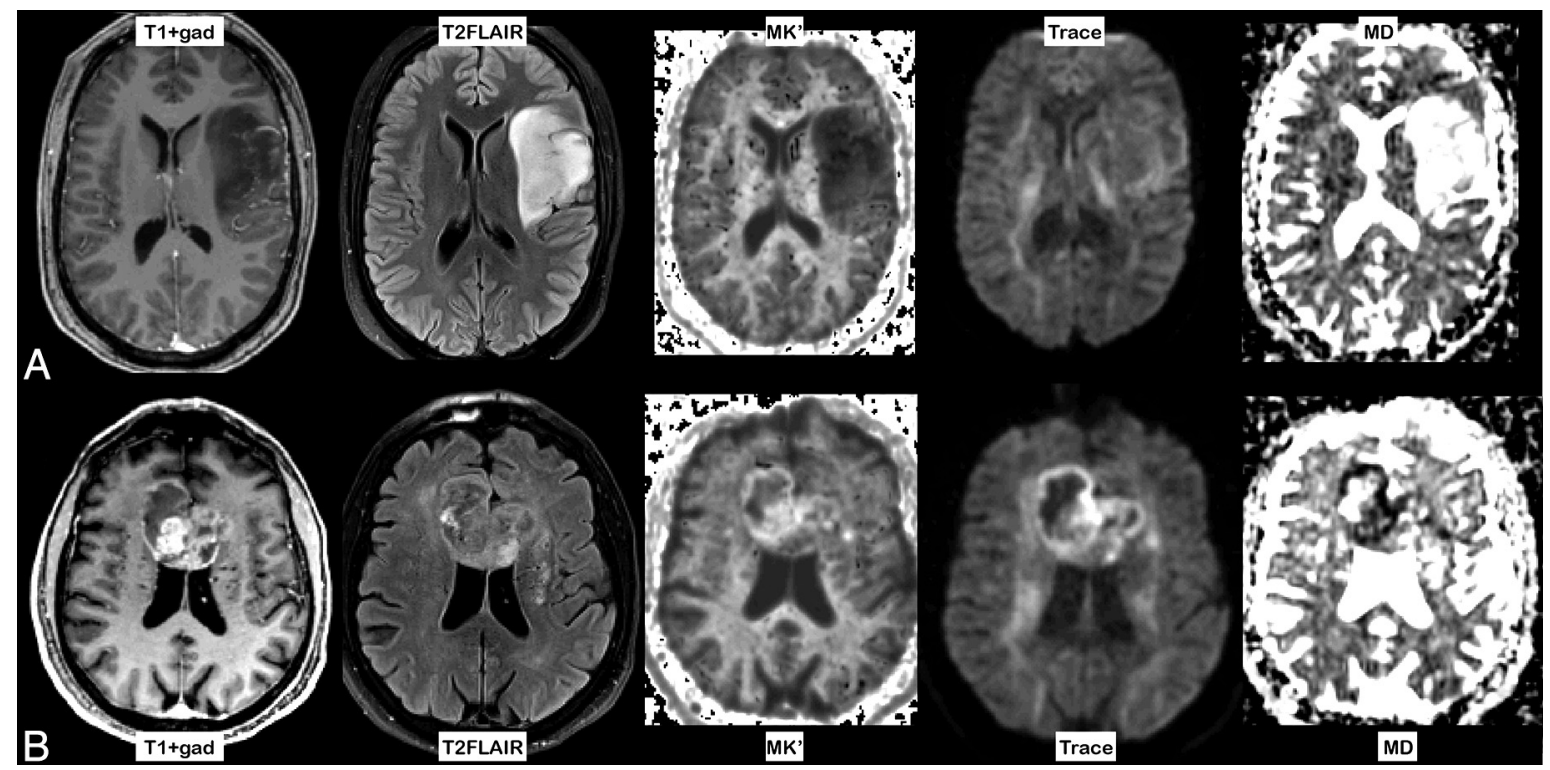

FIG 2. A, Contrast-enhanced T1-weighted and T2 FLAIR images of a grade II astrocytoma grade in the left hemisphere. The map of MK' demonstrates low $\mathrm{MK}^{\prime}$, whereas the trace image and the MD map show high diffusivity (high signal changes on MD). B, Typical example of a glioblastoma, with contrast enhancement on postcontrast T1-weighted and complex signal changes on T2 FLAIR images. Increased MK' is noted in most of the tumor. The trace image and MD show restricted diffusion, primarily in the periphery of the lesion.
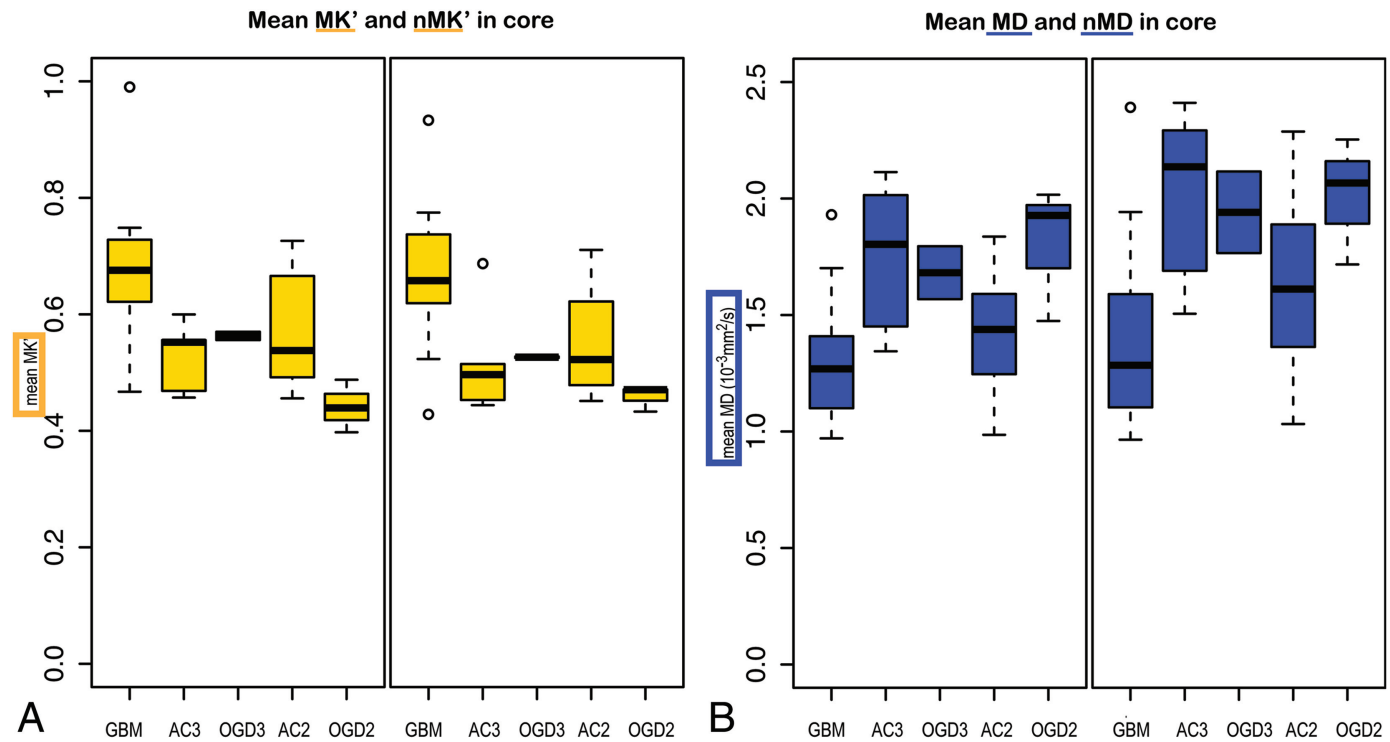

FIG 3. Boxplots of average $M K^{\prime}$ and normalized $M K^{\prime}$ (in yellow) ( $A$ ) and MD and normalized MD (in blue) (B) in the contrast-enhancing tumor core of all patients, grouped according to tumor types and grades, are shown. The horizontal lines in the boxes are the median values, the upper and lower box edges are the 25th and 75th percentiles, respectively, and the upper and lower whiskers represent the minimums and maximums, respectively. AC indicates grade III astrocytoma; ODG3, grade III oligodendroglioma; AC2, grade II astrocytoma; ODG2, grade II oligodendroglioma.

Table 1: Significance of mean $\mathrm{MK}^{\prime}, \mathrm{nMK}^{\prime}, \mathrm{MD}$, and $\mathrm{nMD}$ values in different tumor grades $^{\mathrm{a}}$

\begin{tabular}{lclll}
\hline Tumor Type & MK' $^{\prime}$ & nMK $^{\prime}$ & MD & nMD \\
\hline HGG/LGG & $.028^{\mathrm{b}}$ & .058 & .383 & .511 \\
GBM/all & $<.001^{\mathrm{b}}$ & $.002^{\mathrm{b}}$ & $.006^{\mathrm{b}}$ & $.006^{\mathrm{b}}$ \\
GBM/HGG & $.003^{\mathrm{b}}$ & $.011^{\mathrm{b}}$ & $.004^{\mathrm{b}}$ & $.004^{\mathrm{b}}$ \\
AC3/all & .135 & .135 & .063 & $.033^{\mathrm{b}}$ \\
AC3/HGG & $.011^{\mathrm{b}}$ & $.031^{\mathrm{b}}$ & $.024^{\mathrm{b}}$ & $.019^{\mathrm{b}}$ \\
\hline
\end{tabular}

Note:-AC3 indicates grade III astrocytoma.

a Mean $\mathrm{MK}^{\prime}, \mathrm{nMK}^{\prime}, \mathrm{MD}$, and $\mathrm{nMD}$ values in the tumor core were compared between different tumor grades, and the resulting $P$ values are listed.

${ }^{\mathrm{b}} \mathrm{A} P$ value of $<.05$ indicates a significant difference between groups.
GBMs and the remaining gliomas were higher for $\mathrm{MK}^{\prime}$ and $\mathrm{nMK}^{\prime}$ than for the corresponding MD values. When identifying grade III $\mathrm{AC}$ cases, $\mathrm{MK}^{\prime}$ and MD performed similarly. Areas under the curve calculated from values in the FLAIR masks were either low or not statistically significant. Combining $\mathrm{MK}^{\prime}$ and $\mathrm{MD}$ or $\mathrm{nMK}^{\prime}$ and nMD could increase accuracy, but only $\mathrm{MK}^{\prime}$ and $\mathrm{nMK}^{\prime}$ contributed to the logistic regression model differentiating HGGs from LGGs or identifying GBMs in the entire glioma group. Area under the curve values are given in Table 2. GBMs were diagnosed with $87 \%$ sensitivity and $74 \%$ specificity with a cutoff $\mathrm{MK}^{\prime}$ value of 0.58 ( $87 \%$ sensitivity and $74 \%$ specificity for an $\mathrm{nMK}^{\prime}$ value of 
Table 2: Results of regression analysis ${ }^{\mathrm{a}}$

\begin{tabular}{|c|c|c|c|c|c|c|c|c|c|c|c|c|}
\hline Tumor Type & MK' & $P$ & $\mathrm{nMK}^{\prime}$ & $P$ & MD & $P$ & $\mathrm{nMD}$ & $P$ & $M K^{\prime}+M D$ & $P^{\mathrm{b}}$ & $n M^{\prime}+n M D$ & $P^{\mathrm{b}}$ \\
\hline HGG/LGG & 0.731 & $.045^{c}$ & 0.701 & .056 & 0.595 & .512 & 0.572 & .456 & 0.754 & $.032^{c}$ & 0.746 & $.041^{c}$ \\
\hline GBM/all & 0.842 & $.004^{c}$ & 0.811 & $.004^{c}$ & 0.775 & $.017^{c}$ & 0.772 & $.014^{c}$ & 0.842 & $\begin{array}{l}.220 \\
.045^{c} \\
.796\end{array}$ & 0.807 & $\begin{array}{l}.213 \\
.047^{c} \\
.835\end{array}$ \\
\hline GBM/HGG & 0.886 & $.027^{c}$ & 0.838 & $.022^{c}$ & 0.876 & $.018^{c}$ & 0.876 & $.019^{c}$ & 0.895 & $\begin{array}{l}.127 \\
.204\end{array}$ & 0.905 & $\begin{array}{l}.181 \\
.184\end{array}$ \\
\hline AC3/all & 0.717 & .146 & 0.717 & .170 & 0.766 & .052 & 0.800 & .060 & 0.793 & $\begin{array}{l}.744 \\
.165\end{array}$ & 0.779 & $\begin{array}{l}.885 \\
.155\end{array}$ \\
\hline AC3/HGG & 0.871 & $.039^{c}$ & 0.824 & .055 & 0.835 & $.038^{c}$ & 0.847 & $.042^{c}$ & 0.882 & $\begin{array}{l}.127 \\
.328\end{array}$ & 0.871 & $\begin{array}{l}.301 \\
.265\end{array}$ \\
\hline
\end{tabular}

Note:-AC3 indicates grade III astrocytoma.

${ }^{a}$ The areas under the receiver operating characteristic curves as a measure for diagnostic accuracy are reported.

b Two $P$ values occur when two values (MK' and MD) are tested at the same time.

${ }^{c}$ The variable contributes significantly to the regression model if the corresponding $P$ value is $<.05$.

$0.60)$, whereas the corresponding sensitivity and specificity for an MD value of 1.460 were $80 \%$ and $63 \%$, respectively ( $80 \%$ sensitivity and $63 \%$ specificity for an nMD value of 1.683 ).

\section{DISCUSSION}

In this study, we investigated $\mathrm{MK}^{\prime}$ in the tumor core and the perifocal hyperintensity on T2 FLAIR images in different glioma grades. Our data were acquired with a new and rapid DKI method $^{9}$ that is easily implemented and provides fast acquisition and postprocessing times, which make it potentially useful in clinical departments and for larger studies. The discrimination between HGGs and LGGs was possible only by means of $\mathrm{MK}^{\prime}$, and the $\mathrm{MK}^{\prime}$ and MD values allowed us to identify cases of GBM in the HGG group and in the entire study population. Our receiver operating characteristic analyses revealed high accuracy for $\mathrm{MK}^{\prime}$ and $n \mathrm{nK}^{\prime}$, represented by high areas under the curve. Although we chose to correct MD for the non-Gaussian phenomena (described in "Materials and Methods") to obtain more accurate measurements, we found that $\mathrm{MK}^{\prime}$ was still superior to MD in diagnosing GBMs when evaluating a group of mixed glioma types, whereas $\mathrm{MD}$ and $\mathrm{MK}^{\prime}$ perform similarly when analyzing only HGGs. Moreover, we showed that $\mathrm{MK}^{\prime}$ data variation was significantly smaller in NAWM and larger in NAGM. The high b-values, used for DKI, dampen the signal considerably, and with noise being constant, the signal-to-noise ratio decreases. Because MD is higher in NAGM than in NAWM, the decrease in signal-to-noise ratio is expected to be more distinct in NAGM, negatively impacting parameter estimates and causing larger variation.

Currently, few studies in which DKI parameters in patients with glioma were investigated have been published. Van Cauter et $\mathrm{al}^{7}$ examined 28 patients (17 with an HGG and 11 with an LGG, both oligodendroglial and astrocytic types) with a conventional DKI sequence taking 17 minutes 29 seconds and showed a significant difference in $\mathrm{MK}, \mathrm{nMK}$, and $\mathrm{nMD}$ values between HGGs and LGGs, with areas under the curve of $>0.8$ for all 3 values. Median values in the tumor core, the perifocal hyperintensity on T2 FLAIR images, and the contralateral NAGM were similar to ours, but the median value for the contralateral NAWM was considerably lower than ours. ${ }^{7}$ The higher MK values for NAWM measured in our study corresponds better, however, to values reported earlier. ${ }^{2,9}$ Values for single tumor grades (eg, GBM or grade III AC) were not reported by these studies. Raab et $\mathrm{al}^{6}$ evaluated 33 patients with an astrocytoma only (AC grade 2, AC grade
3, or GBM) by using a DKI sequence that took 11 minutes 57 seconds. Mean MK values in the core of grade II ACs, grade III ACs, and GBMs were somewhat higher in their study (between 0.09 higher for $\mathrm{nMK}$ in grade III ACs and 0.14 for MK in GBMs), and their ability to separate tumor grades was slightly better than ours. Several possible causes for these discrepancies are conceivable, including unconfirmed histopathology in 4 of our cases, previous surgery, ${ }^{3}$ the risk of misdiagnosis caused by imprecise sampling in cases of biopsy, the inclusion of gliomas with both oligodendroglial and astrocytic histology results, differences between the MR imaging systems used for data acquisition (particularly gradient performance and its influence on diffusion times and achievable echo times), different technical and postprocessing approaches, and the distinct physical nature of MK and MK'.

We were able to differentiate HGGs from LGGs by means of MK', but our separation of GBMs and AC3s from the remaining gliomas might be clinically even more critical than the rough classification of HGGs and LGGs that in most cases is accomplished by conventional or perfusion-weighted MR imaging. ${ }^{12}$ Even more detailed subtyping of gliomas in those with a relatively favorable outcome and those with a poor outcome are becoming increasingly relevant, because different therapeutic approaches can be considered. The prediction of patient outcome by means of imaging biomarkers correlating them with genomic subtypes is an expanding field, and MK should be included in the future. ${ }^{13} \mathrm{We}$ also suggest that DKI might be an important diagnostic tool for assessing tumor progression and treatment response. It is well known that LGGs progress to higher grades with time, a process that is essential to monitor for accurately timed treatment adjustment, and gradually increasing MK might be a valuable tool for detecting these subtle changes in dedifferentiation. Therefore, visualization of aggressive regions within a lesion by detailed MK mapping could have important implications on the neurosurgical approach or radiation treatment planning. A promising additional application area for DKI is response evaluation of patients during antiangiogenic treatment, which is currently a diagnostic challenge. ${ }^{14}$ Our fast DKI protocol with very short postprocessing times enables real-time image reconstruction on the scanner and is therefore a promising tool for integrating multimodal evaluation of patients with glioma.

Only a small number of patients were included in this study, which might limit its power but can also be regarded as a strength, 
because we still showed a significant difference between tumor grades. We aimed to compare our new method with that of 2 previously published studies in which a data volume of approximately the same scale was investigated, and we could reproduce most of their results with a fast sequence that maps $\mathrm{MK}^{\prime}$ on the basis of only 13 images ( 2 minutes 46 seconds in our case) and a DKI postprocessing time of just a few seconds. Other metrics that evaluate directional non-Gaussianity, such as axial or radial kurtosis, have been described and may be of interest, because they are likely to reveal different and supplemental information about microstructure. ${ }^{15}$ These kurtosis metrics cannot be estimated from the data, because they are derived from the full kurtosis tensor, and reliable estimation of the tensor would require the measurement of additional diffusion directions and the nonlinear fitting of the results to a diffusion model. These requirements would add considerably to the scanning and postprocessing times, which would be particularly inexpedient in clinical applications. As a result, the significance of these measures for tumor grading was not evaluated in this study. Axial and radial kurtosis were assessed by Van Cauter et al, ${ }^{7}$ but the diagnostic impact of these parameters did not differ considerably from the MK in their study. A limitation of our study is that some of the patients with GBM received steroid treatment at the time of imaging. Steroids might have an influence on $\mathrm{MK}, \mathrm{MK}^{\prime}$, and $\mathrm{MD}$ values by reducing the amount of edema. We are not able to correct for this effect. Finally, potential partial volume effects caused by co-registration steps might have influenced the results. We inspected our data for co-registration errors closely and adjusted them thoroughly to avoid the inclusion of CSF and necrosis, but inexactness was still possible.

The increasing MK in GBM is interesting with regard to the underlying microstructural characteristics of these tumors. Little is known about what might cause MK alterations, but it has been postulated that changes in the quantity of cell membranes and the amount of intracellular and extracellular protein are likely to influence the degree of non-Gaussianity. ${ }^{16}$ The chaotic vascular architecture of and micronecrosis in GBMs might contribute further to the microstructural complexity causing an increase in MK. The association between increased cellularity and restricted water diffusion, estimated by MD, was investigated in several previous studies, ${ }^{17-19}$ but the utility of this parameter was not always reconfirmed. ${ }^{20,21} \mathrm{~A}$ weak correlation can be a result of concomitant MD increases caused by edema and micronecrosis in HGGs, and it remains to be shown if MK can add important diagnostic information. MK calculated from a conventional DKI sequence and MK' obtained by our fast method are distinct quantities, because they are estimated in a different way. However, it was shown that they are highly correlated both in normal brain ${ }^{9}$ and in acute stroke tissue. ${ }^{22}$ We are not able to compare both approaches, because only the rapid version was acquired, but we expect this correlation to be the same in our patients and avail ourselves of the comparison with literature values.

\section{CONCLUSIONS}

We have shown that $\mathrm{MK}^{\prime}$ is a valuable tool for glioma grading and that our fast DKI method provides robust measurements comparable to those from the literature. These results enable larger-scale implementation of DKI in clinical studies and allow for the investigation of its significance in treatment evaluation, diagnosis of LGG dedifferentiation, and detection of recurrent disease. Moreover, imaging with a rapid DKI technique in clinically unstable patients, such as those after a stroke or trauma, has the potential to provide new insights into the pathophysiology and longitudinal progression of these diseases. ${ }^{22-25}$

\section{ACKNOWLEDGMENTS}

We thank Kim Mouridsen for his advice on the statistical analysis. B.H. acknowledges support from NIH 1R01EB012874-01 and Aarhus University's nanomedicine center (LUNA).

Disclosures: Anna Tietze—RELATED: Grant: Danish Ministry of Science, Technology and Innovation's University Investment Grant (MINDLab).* Mikkel Hansen-RELATED: Grant: Danish Ministry of Science, Technology and Innovation*; UNRELATED: Stock/Stock Options: Combat Stroke, Comments: co-founder, shareholder, and member of the board (unpaid) of the spin-out company named "Combat Stroke." Lief Østergaard—RELATED: Grant: Danish Ministry for Research and Innovation.* Sune Nørhøj Jespersen—UNRELATED: Grants/Grants Pending: The Lundbeck Foundation, Comments: 2,000,000 DKK to a project on biological interpretations of diffusion imaging (mainly postdoctoral salary); Patents (planned, pending, or issued): patent pending on the fast-diffusion kurtosis method used in the study and 2 related to measurements of capillary blood flow heterogeneity. Siemens has shown limited interest in the first patent. Torben E. Lund-RELATED: Grant: Danish Agency for Science, Technology and Innovation,* Comments: Aarhus University received $120,000,000$ DKK to the UNIK project MINDLab. B. Hansen—RELATED: Grant: salary and work supported by the Danish Ministry of Science, Technology and Innovation's University Investment Grant (MINDLab).* My work was also supported by National Institutes of Health 1R01EB012874-01.* *Money paid to the institution.

\section{REFERENCES}

1. Gerstner ER, Sorensen AG. Diffusion and diffusion tensor imaging in brain cancer. Semin Radiat Oncol 2011;21:141-46

2. Jensen JH, Helpern JA, Ramani A, et al. Diffusional kurtosis imaging: the quantification of non-Gaussian water diffusion by means of magnetic resonance imaging. Magn Reson Med 2005;53:1432-40

3. Zhuo J, Xu S, Proctor JL, et al. Diffusion kurtosis as an in vivo imaging marker for reactive astrogliosis in traumatic brain injury. Neuroimage 2012;59:467-77

4. Veraart J, Poot DH, Van Hecke W, et al. More accurate estimation of diffusion tensor parameters using diffusion kurtosis imaging. Magn Reson Med 2011;65:138-45

5. Steven AJ, Zhuo J, Melhem ER. Diffusion kurtosis imaging: an emerging technique for evaluating the microstructural environment of the brain. AJR Am J Roentgenol 2014;202:W26-33

6. Raab P, Hattingen E, Franz K, et al. Cerebral gliomas: diffusional kurtosis imaging analysis of microstructural differences. Radiology 2010;254:876-81

7. Van Cauter S, Veraart J, Sijbers J, et al. Gliomas: diffusion kurtosis MR imaging in grading. Radiology 2012;263:492-501

8. Lu H, Jensen JH, Ramani A, et al. Three-dimensional characterization of non-Gaussian water diffusion in humans using diffusion kurtosis imaging. NMR Biomed 2006;19:236-47

9. Hansen B, Lund TE, Sangill R, et al. Experimentally and computationally fast method for estimation of a mean kurtosis. Magn Reson Med 2013;69:1754-60

10. Hansen B, Lund TE, Sangill R, et al. Erratum: Experimentally and Computationally Fast Method for Estimation of a Mean Kurtosis. Magn Reson Med 2014;71:2250

11. Jensen JH, Hu C, Helpern JA. Rapid data acquisition and postprocessing for diffusional kurtosis imaging. In: 17th Annual Meeting of ISMRM, Honolulu, Hawaii. April 18-24, 2009

12. Lev MH, Ozsunar Y, Henson JW, et al. Glial tumor grading and outcome prediction using dynamic spin-echo MR susceptibility mapping compared with conventional contrast-enhanced MR: 
confounding effect of elevated rCBV of oligodendrogliomas [corrected]. AJNR Am J Neuroradiol 2004;25:214-21

13. Jain R, Poisson LM, Gutman D, et al. Outcome prediction in patients with glioblastoma by using imaging, clinical, and genomic biomarkers: focus on the nonenhancing component of the tumor. Radiology 2014;272:484-93

14. Ellingson BM, Cloughesy TF, Lai A, et al. Quantitative volumetric analysis of conventional MRI response in recurrent glioblastoma treated with bevacizumab. Neuro Oncol 2011;13:401-09

15. Carmeliet P, De Smet F, Loges S, et al. Branching morphogenesis and antiangiogenesis candidates: tip cells lead the way. Nat Rev Clin Oncol 2009;6:315-26

16. Maier SE, Sun Y, Mulkern RV. Diffusion imaging of brain tumors. NMR Biomed 2010;23:849-64

17. Hilario A, Ramos A, Perez-Nunez A, et al. The added value of apparent diffusion coefficient to cerebral blood volume in the preoperative grading of diffuse gliomas. AJNR Am J Neuroradiol 2012;33:701-07

18. Khayal IS, Polley MY, Jalbert L, et al. Evaluation of diffusion parameters as early biomarkers of disease progression in glioblastoma multiforme. Neuro Oncol 2010;12:908-16

19. Moffat BA, Chenevert TL, Lawrence TS, et al. Functional diffusion map: a noninvasive MRI biomarker for early stratification of clinical brain tumor response. Proc Natl Acad Sci U S A 2005;102:5524-29

20. Castillo M, Smith JK, Kwock L, et al. Apparent diffusion coefficients in the evaluation of high-grade cerebral gliomas. AJNR Am J Neuroradiol 2001;22:60-64

21. Rose S, Fay M, Thomas P, et al. Correlation of MRI-derived apparent diffusion coefficients in newly diagnosed gliomas with $\left[{ }^{18} \mathrm{~F}\right]-$ fluoro-L-dopa PET: what are we really measuring with minimum ADC? AJNR Am J Neuroradiol 2013;34:758-64

22. Sun PZ, Wang Y, Mandeville E, et al. Validation of fast diffusion kurtosis MRI for imaging acute ischemia in a rodent model of stroke. NMR Biomed 2014;27:1413-18

23. Taoka T, Fujioka M, Sakamoto M, et al. Time course of axial and radial diffusion kurtosis of white matter infarctions: period of pseudonormalization. AJNR Am J Neuroradiol 2014;35:1509-14

24. Hori M, Fukunaga I, Masutani Y, et al. Visualizing non-Gaussian diffusion: clinical application of $q$-space imaging and diffusional kurtosis imaging of the brain and spine. Magn Reson Med Sci 2012;11:221-33

25. Jensen JH, Falangola MF, Hu C, et al. Preliminary observations of increased diffusional kurtosis in human brain following recent cerebral infarction. NMR Biomed 2011;24:452-57 